\title{
The Potential of Tidal Power from the Bay of Fundy
}

\author{
Justine M. McMillan ^ AND Megan J. Lickley \\ Department of Mathematics \& Statistics, Acadia University, Wolfville, NS, Canada \\ Advisors \\ Richard Karsten AND Ronald Haynes
}

\begin{abstract}
Large tidal currents exist in the Minas Passage, which connects the Minas Basin to the Bay of Fundy off the north-western coast of Nova Scotia. The strong currents through this deep, narrow channel make it a promising location for the generation of electrical power using in-stream turbines. Using a finite-volume numerical model, the high tidal amplitudes throughout the Bay of Fundy are simulated within a root mean square difference of $8 \mathrm{~cm}$ in amplitude and $3.1^{\circ}$ in phase. The bottom friction in the Minas Passage is then increased to simulate the presence of turbines and an estimate of the extractable power is made. The simulations suggest that up to $6.9 \mathrm{GW}$ of power can be extracted; however, as a result, the system is pushed closer to resonance which causes an increase in tidal amplitude of over $15 \%$ along the coast of Maine and Massachusetts. The tides in the Minas Basin will also experience a decrease of $30 \%$ in amplitude if the maximum power is extracted. Such large changes can have harmful environmental impacts; however, the simulations also indicate that up to $2.5 \mathrm{GW}$ of power can be extracted with less than a $6 \%$ change in the tides throughout the region. According to Nova Scotia Energy, 2.5 GW can power over 800,000 homes.
\end{abstract}

\section{Introduction}

The highest tides in the world occur in the Bay of Fundy, which is located between Nova Scotia and New Brunswick (see Figure 1). In the open ocean, tides typically have ranges of one to two metres [1]; however, the difference between high and low tide in the Minas Basin can exceed $16 \mathrm{~m}$ [1]. As discussed in Section 2, the large tidal amplitudes in this

*Corresponding author: Department of Mathematics \& Statistics Acadia University, Wolfville, NS, B4P 2R6, Canada. (email: mcmillanjustine@gmail.com) 
region are driven by the near-resonance of the Bay of Fundy - Gulf of Maine system, which has a natural period of approximately 13 hours [5, 9] close to the 12.42 hour period of the forcing tides.

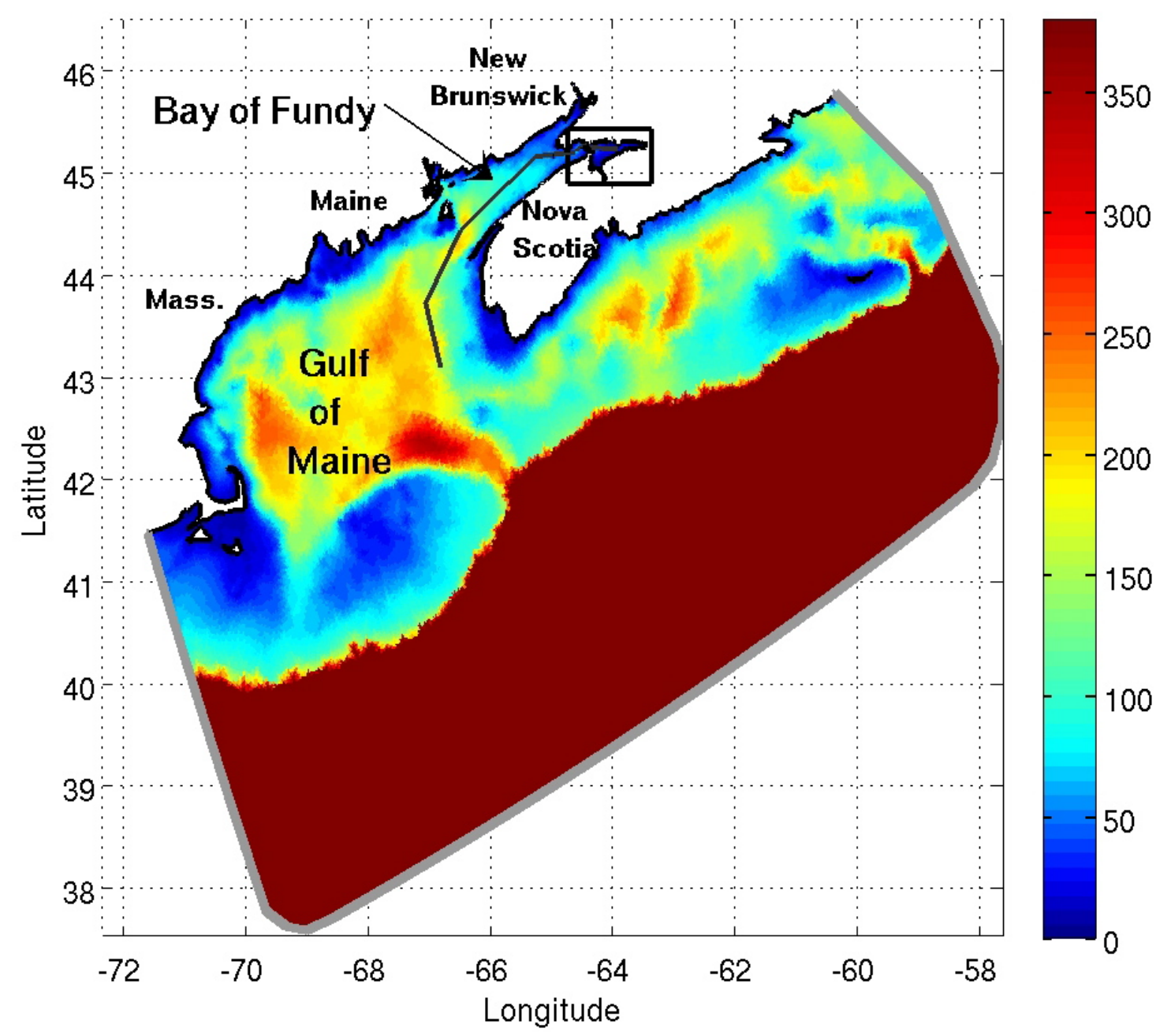

Figure 1: The Gulf of Maine - Bay of Fundy region over which the tides were numerically simulated. The colours represent the bathymetry $(\mathrm{m})$ of the region, but it should be noted that beyond the continental shelf (the dark red region) the depth is typically in excess of $4000 \mathrm{~m}$. The input tides are specified on the open boundary of the domain which is illustrated by the thick gray line, whereas the black line passing through the center of the Bay of Fundy approximates the path of the tidal wave. The rectangle encloses the Minas Passage and Minas Basin region which is displayed in Figure 3.

The rise and fall of a large body of water suggests that the potential energy is high in the Bay of Fundy. A large volume flux is required for these significant changes in amplitude to occur, therefore fast currents arise in narrow channels which results in high kinetic energy. Greenberg [9] calculates both potential and kinetic energies on the order of $10^{14} \mathrm{~J}$. Until recently, the methods of harvesting this tidal energy were limited to capturing water in a dam at high tide and generating electricity by releasing the water at low tide. Previous studies [9, 12, have investigated the possibility of building such a dam near the Minas Passage. Both studies used numerical simulations to conclude that 
the tides would increase significantly along the coast of Maine and Massachusetts, as a result of pushing the system closer to resonance. In particular, Greenberg [9] estimated a $15 \mathrm{~cm}$ increase in amplitude in Boston.

Now, with stronger demands for green energy and the development of new technology, the prospect of tidal power has resurfaced. In-stream turbines have recently been developed and proposals have been made for their implementation into regions of high tidal flow. Plans are currently underway for the installation of three turbines in the Minas Passage [11]. Ideally, these turbines, which operate much like wind turbines, would produce a predictable, renewable source of power with less of an impact on the environment than a dam. Although similar to wind turbines, the dynamics of large scale power extraction from a channel like the Minas Passage has important differences (see discussion in [6, 7]). First, the restriction of the channel forces flow through the turbines and, second, the placement of turbines in the channel will impact the tides in the Minas Basin and throughout the Bay of Fundy.

Garrett and Cummins [6, 7] suggest a simple power estimate, based on only the kinetic energy flux, is not accurate in this situation because the turbines cause the flow through the channel to decrease. As a result, the time lag between high tide across the channel increases, which causes the pressure gradient to increase. Since it is this pressure gradient that drives the flow through the channel, the maximum extractable power is actually greater than the kinetic energy flux. Karsten et al. [10] adapt the theory of Garrett and Cummins [6, 7] to the Minas Passage and provide an assessment of the tidal current energy in the region. Here, we attempt to put the conclusions of [10] into context by presenting the results in a more accessible format. We also give a more detailed description of basic tidal theory in an attempt to explain the dynamics of the Bay of Fundy system.

More specifically, we begin in Section 2, by presenting a simple description of the resonance that generates the high tides in the Bay of Fundy. Section 3 then contains a description of the numerical model that was used to simulate the tides and we illustrate that our results are accurate by making comparisons to measured tidal data. The most significant results of our research are summarized in Section 4, where an estimate of the maximum extractable power is made both theoretically and numerically. We also examine both the near-field and far-field effects of extracting such a large quantity of energy from a resonant system. We then conclude the paper by attempting to estimate the amount of power that can be extracted with an acceptable change in the tides.

\section{Tidal Wave Resonance}

Equilibrium tidal theory predicts that tides originate from the gravitational forces of the Moon and the Sun acting on the world's oceans. In particular, the Moon exerts a force on the Earth causing it to accelerate towards the Moon; however, because the oceans on the side facing the Moon are closer than the Earth, they experience a greater acceleration. Similarly, on the distant side of the Earth, the Earth accelerates faster than the ocean, creating a second aqueous bulge. It takes 24.84 hours for the Earth to complete a single rotation relative to the Moon, hence, the semidiurnal lunar constituent of the tide, $\mathrm{M}_{2}$, has a period of 12.42 hours.

Other tidal constituents, which depend on the gravitational force of the Sun, the tilt 
of the Earth's rotation axis, and the elliptical orbits of the astronomical bodies, have varying periods. In general, the proximity of the Moon to the Earth causes the $\mathrm{M}_{2}$ tidal constituent to dominate the tidal forcing; however, the bathymetry in a region also plays an important role in determining the amplitude and period of the tides.

It is indeed the bathymetry and geometry of the Bay of Fundy that cause the amplification of the $\mathrm{M}_{2}$ tide in this region. The presence of the continental shelf, the average depth and the length of the combined Bay of Fundy and Gulf of Maine are all factors causing the natural period of the Bay of Fundy to be slightly greater than 12.42 hours.

An estimate of this natural period can be determined by analyzing the solution to a one-dimensional gravity wave equation in a finite-channel, which is given by the following partial differential equation and boundary conditions as found in [8]:

$$
\begin{aligned}
& \frac{\partial^{2} \zeta}{\partial t^{2}}=c^{2} \frac{\partial^{2} \zeta}{\partial x^{2}}, \quad 0<x<L \\
& \zeta(0, t)=\zeta_{0} \cos (\omega t), \\
& \frac{\partial \zeta}{\partial x}(L, t)=0
\end{aligned}
$$

where $L$ is the length of the channel, $\zeta=\zeta(x, t)$ is the surface elevation, $\omega$ is the radian frequency of the elevation and $c$ is the speed of a gravity wave given by, $c=\sqrt{g H}$, where $g$ is the gravitational acceleration and $H$ is the depth of the channel. The boundary conditions given by Equations (2) and (3) respectively ensure that the elevation at the opening of the channel follows the forcing tide and that there is no flux through the closed end of the channel. The general solution to Equation (1) has the following form of a traveling wave,

$$
\zeta(x, t)=A \cos [k(x-L)-\omega t]+B \cos [k(x-L)+\omega t],
$$

where $k=\omega / c$ is the wave number. The solution given by Equation (4) represents an incident wave traveling in one direction and a reflected wave traveling in the opposite direction. By applying the boundary conditions given by Equations (2) and (3), the coefficients are found to be

$$
A=B=\frac{\zeta_{0}}{\cos (k L)}
$$

The resulting solution to the differential equation is

$$
\zeta(x, t)=\zeta_{0} \frac{\cos [k(x-L)] \cos (\omega t)}{\cos (k L)} .
$$

Resonance occurs when the elevation of the tides at the closed end of the channel is maximized with respect to the elevation at the channel opening. This ratio, given by

$$
\frac{\zeta(L, t)}{\zeta(0, t)}=\frac{1}{\cos (k L)},
$$


will approach infinity if $k L=\left(n+\frac{1}{2}\right) \pi$, therefore the natural modes of the Bay of Fundy correspond to the following frequencies,

$$
\omega_{n}=\left(n+\frac{1}{2}\right) \pi \frac{\sqrt{g H}}{L}, \quad \text { where } n=0,1,2, \ldots
$$

These frequencies are the eigenvalues of the boundary value problem given by Equations (1) - (3).

If the length and depth of the Bay of Fundy are estimated as $L=400 \mathrm{~km}$ and $H=110 \mathrm{~m}$, then the natural modes corresponding to $n=0,1,2$ and 3 , have respective periods $\left(T_{n}=\frac{2 \pi}{\omega_{n}}\right)$ of $T_{n}=13.5,4.5,2.7$, and 1.9 hours. The $\mathrm{M}_{2}$ tidal constituent has a period of 12.42 hours; therefore, it can be concluded that only the $n=0$ mode resonates with the forcing tide, making 13.5 hours the approximate natural period of the Bay of Fundy. In comparison, a natural period of 12.85 hours is estimated using numerical simulations (see Section 4). This one-dimensional theory, and in particular Equation (5), can also be used to predict an amplitude of $7.1 \mathrm{~m}$ in the Minas Passage by noting that the amplitude of the tide at the opening of the channel is about $1.0 \mathrm{~m}$. As Figure 3 illustrates, the numerical simulations give an amplitude of $7 \mathrm{~m}$ in the Minas Basin; therefore, although this simple theory does not take into account the changing depth of the ocean, bottom friction or the nonlinear effects of the flow, it can be used to describe basic tidal resonance.

Due to the resonance described above, the $\mathrm{M}_{2}$ tidal constituent is significantly amplified in the Bay of Fundy. In [4, Dupont et al. conclude that the amplitude of the $\mathrm{M}_{2}$ tide is over 4.5 times greater than any other tidal constituent in the Bay of Fundy; therefore, only the $\mathrm{M}_{2}$ tide was used to force our numerical model. Slightly more accurate tides could have been achieved by including the less dominant constituents in the model; however, longer simulations would have been needed because these constituents primarily influence the monthly and yearly variations in the tides.

\section{$3 \quad$ Numerically Modelling the Tides}

To numerically simulate the tides, a finite element grid was used which consisted of the Bay of Fundy, Gulf of Maine and a region of the Atlantic Ocean as illustrated in Figures 1 and 2. The entire region (Figure 1) was approximately $600000 \mathrm{~km}^{2}$ in area. The original grid, which was obtained from David Greenberg at the Bedford Institute of Oceanography, consisted of 9521 non-uniform triangular elements; however, we increased the resolution by dividing each element in the original grid into four similar triangles. The new nodes were located at the midpoints of the sides of the original triangles with values for the ocean depth determined using linear interpolation. This process was again repeated creating a third grid with the number of triangular elements increased by a factor of 16 in comparison to the original grid. Due to the properties of similar triangles, this method of increasing the resolution ensured that the desirable properties of the original grid, such as the angles within the triangles, were all maintained. Numerical simulations were performed using each of the three grids and convergence in the energy and power values were obtained. By increasing the resolution of the grid, we also increased the number of points across the Minas Passage from 5 in the original grid to 20 in the final grid. 


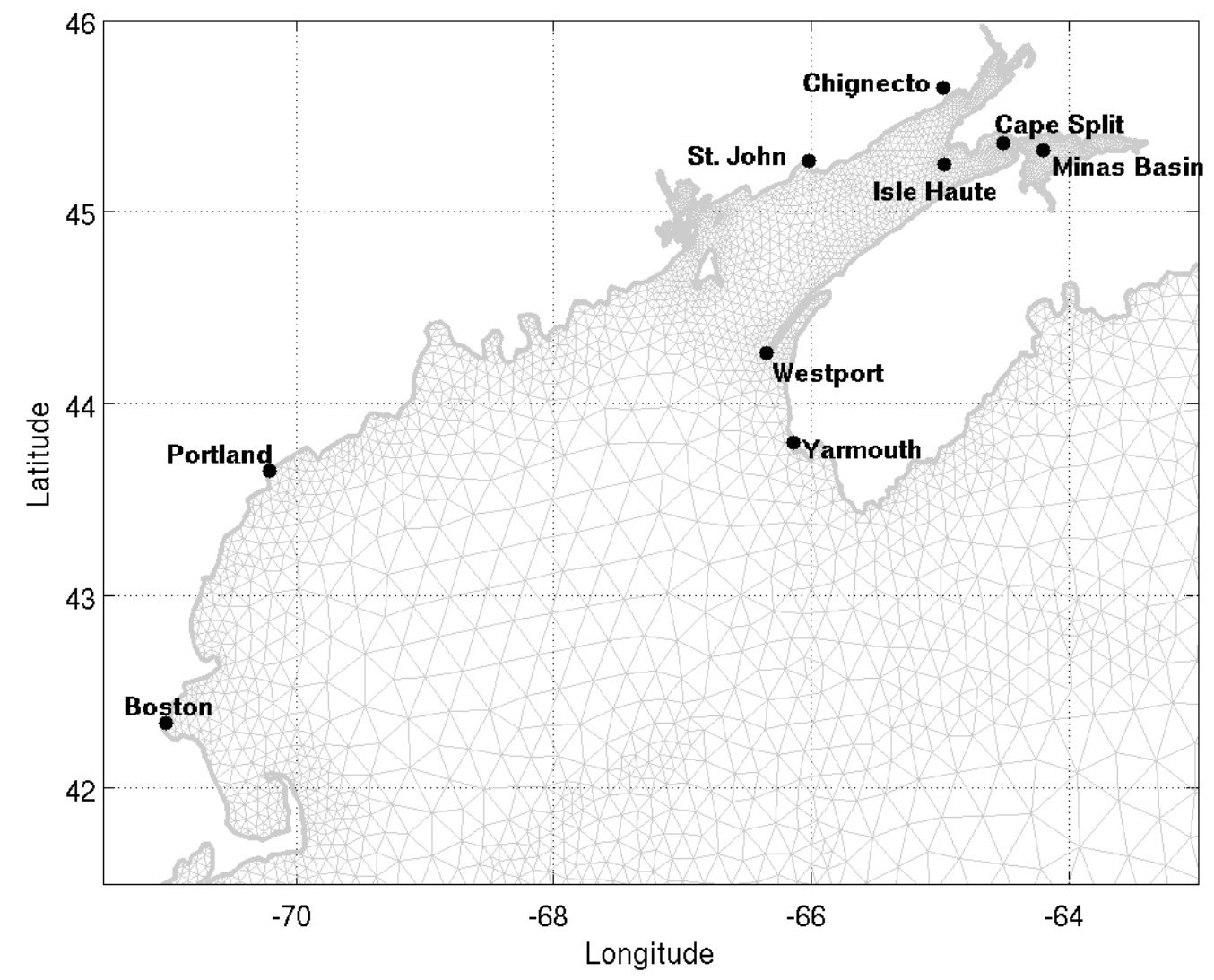

Figure 2: The location of nine observation stations which were used to compare the numerical results to measured data. The figure also displays the finite element grid that was obtained from David Greenberg. For the results presented in this paper, a higher resolution grid was used where each of the triangular elements above was divided into 16 similar triangles.

An important characteristic of the finite element grid, which is evident in Figure 2, is that regions of complex bathymetry and geometry are characterized by a greater resolution. In particular, the densities of the triangular elements near the coastline and in shallow regions are much greater than that in the deep Atlantic Ocean.

To numerically simulate the tides, a Finite-Volume Coastal Ocean Model (FVCOM) [3] is used. For the purposes of this research, the solutions are governed by the twodimensional momentum and continuity equations, given by

$$
\begin{aligned}
& \frac{\partial u}{\partial t}+u \frac{\partial u}{\partial x}+v \frac{\partial u}{\partial y}-f v=-g \frac{\partial \zeta}{\partial x}-F_{x}, \\
& \frac{\partial v}{\partial t}+u \frac{\partial v}{\partial x}+v \frac{\partial v}{\partial y}+f u=-g \frac{\partial \zeta}{\partial y}-F_{y}, \\
& \frac{\partial \zeta}{\partial t}+\frac{\partial}{\partial x}[u(h+\zeta)]+\frac{\partial}{\partial y}[v(h+\zeta)]=0,
\end{aligned}
$$

where $x$ and $y$ are the east and north directions; $u$ and $v$ are the depth integrated east and north velocities; $f$ is the Coriolis parameter $(f=2 \pi \sin$ (latitude) $) ; g$ is the gravitational 
acceleration; $h$ is the undisturbed depth of water; $\zeta$ is the height of the free surface relative to $h ; t$ is the time; and, $F_{x}$ and $F_{y}$ are the east and north quadratic friction forces given by

$$
F_{x}=\kappa u \frac{\sqrt{u^{2}+v^{2}}}{h+\zeta} \text { and } F_{y}=\kappa v \frac{\sqrt{u^{2}+v^{2}}}{h+\zeta}
$$

where $\kappa$ is the bottom friction coefficient.

Each triangular element in the grid, illustrated in Figure 2, is represented by its three nodes. At each of the nodes, the longitude, latitude and ocean depth are specified. The phase and amplitude of the $\mathrm{M}_{2}$ tide is also specified at the nodes located on the open boundary (see Figure 1), which provides the forcing necessary to generate the tides. The model allows water to freely flow into or out of the computational domain along the open boundary; however, it is necessary to include a sponge layer to damp out reflected waves, removing numerical instabilities. It is important that the open boundary is located beyond the continental shelf, allowing the Bay of Fundy - Gulf of Maine system to respond freely to the tidal forcing. Using FVCOM, the tides are then simulated with the values of $u, v$ and $\zeta$ saved every $1 / 24$ of a tidal period for the last four periods of a 16 tidal period run. The amplitude and phase of the tides can then be calculated at each node by fitting a cosine curve to the surface height. To ensure that the simulations were producing accurate results, these values were compared to measured values for the tidal phase and amplitude at 51 observation stations obtained from David Greenberg. The locations of nine of these stations are shown in Figure 2.

In order to achieve the most accurate results, the model was tuned by adjusting the bottom friction coefficient until the mean amplitude difference between the calculated and observed values was a minimum. After conducting several numerical simulations, this bottom friction coefficient was determined to be 0.0026. In comparison, Dupont et al. [4] achieved their smallest error using a coefficient of 0.0025; whereas, Sucsy et al. [12] used 0.002. Greenberg [9], on the other hand, used two different values - 0.0024 in the Gulf of Maine, and 0.0021 for the remainder of the region.

The final results for nine observation stations are summarized in Table1. Observations were not obtained for Cape Split; however, it is included in the table because the phase and amplitude at the entrance to the Minas Passage is important in the discussion of turbines in Section 4. For all 51 stations, the root mean square (rms) amplitude difference is $8 \mathrm{~cm}$ and the rms phase difference is $3.1^{\circ}$. In general, our errors are slightly smaller than those of Greenberg [9] and comparable to Sucsy et al. [12] and Dupont et al. [4].

The calculated tidal amplitudes and phases are displayed for the Minas Passage and Minas Basin in Figure 3. It is evident in this figure that the amplitude of the simulated tide is greater than $6 \mathrm{~m}$ in some regions of the Minas Basin. The large phase lag of $10.1^{\circ}$ between Cape Split and the Minas Basin (Table 1) indicates that there is a large difference in the surface elevation (tidal head) across the channel. It is the hydrostatic pressure associated with this tidal head that forces large tidal currents through the channel. 


\begin{tabular}{|l|rr|rr|rr|}
\hline & \multicolumn{2}{|c|}{ Observed } & \multicolumn{2}{c|}{ Modeled } & \multicolumn{2}{c|}{ Difference } \\
Station & Amp. & Phase & Amp. & Phase & Amp. & Phase \\
\hline Boston & 1.34 & 111 & 1.36 & 116.2 & -0.02 & 5.2 \\
Portland & 1.33 & 103 & 1.36 & 106.4 & -0.03 & 3.4 \\
Saint John & 3.04 & 98 & 3.05 & 97.2 & -0.02 & -0.8 \\
Chignecto & 4.18 & 103 & 4.27 & 100.4 & -0.09 & -2.6 \\
Minas Basin & 5.48 & 121 & 5.35 & 117.9 & 0.13 & -3.1 \\
Cape Split & - & - & 4.71 & 107.8 & - & - \\
Isle Haute & 4.18 & 99 & 4.07 & 96.5 & 0.11 & -2.5 \\
Westport & 2.20 & 80 & 2.18 & 79.1 & 0.02 & -0.9 \\
Yarmouth & 1.63 & 63 & 1.66 & 63.1 & -0.03 & 0.1 \\
\hline
\end{tabular}

Table 1: Observed and calculated amplitudes $(\mathrm{m})$ and phases $\left(^{\circ}\right)$ for several observation stations. There was no measured data for Cape Split.

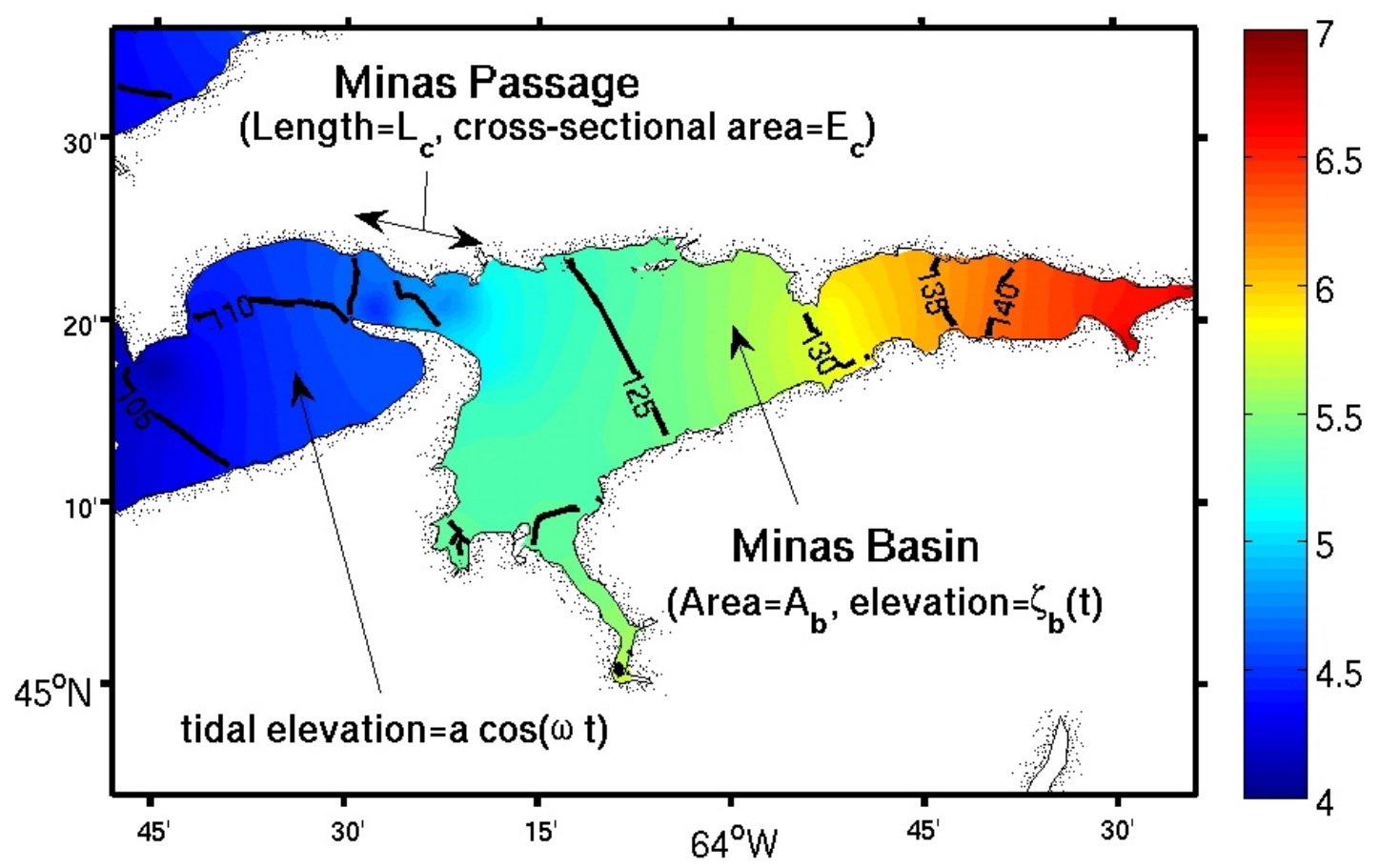

Figure 3: The numerically simulated amplitude $(\mathrm{m})$ and phase $\left(^{\circ}\right)$ of the $\mathrm{M}_{2}$ tide in the Minas Passage and Minas Basin are represented by the colours and contours, respectively. The over $10^{\circ}$ phase difference across the Minas Passage creates the large tidal head driving the flow through the channel. The parameters referred to throughout the text are also displayed.

Because the primary goal of the research was to investigate the extraction of tidal power using in-stream turbines, it was desired to determine the location of maximum power density. The power per unit area can be determined by calculating the time averaged kinetic energy flux which is given by 


$$
P_{K E}=\frac{\rho}{2 T} \int_{0}^{T}\left(u^{2}+v^{2}\right)^{3 / 2} d t
$$

where $T$ is the length of one tidal period (12.42 hours) and $\rho$ is the density of the water. As illustrated by Figure 4, the power density in the Minas Passage exceeds $18 \mathrm{~kW} / \mathrm{m}^{2}$ which is much greater than anywhere else in the region due to the high velocity of the flow passing through this channel. In fact, excluding the areas immediately surrounding the Minas Passage, the average power density is less than $2 \mathrm{~kW} / \mathrm{m}^{2}$. By integrating across the channel, Triton Consultants [14] estimated that the total power associated with the kinetic energy flux is $1.9 \mathrm{GW}$ in the Minas Passage.

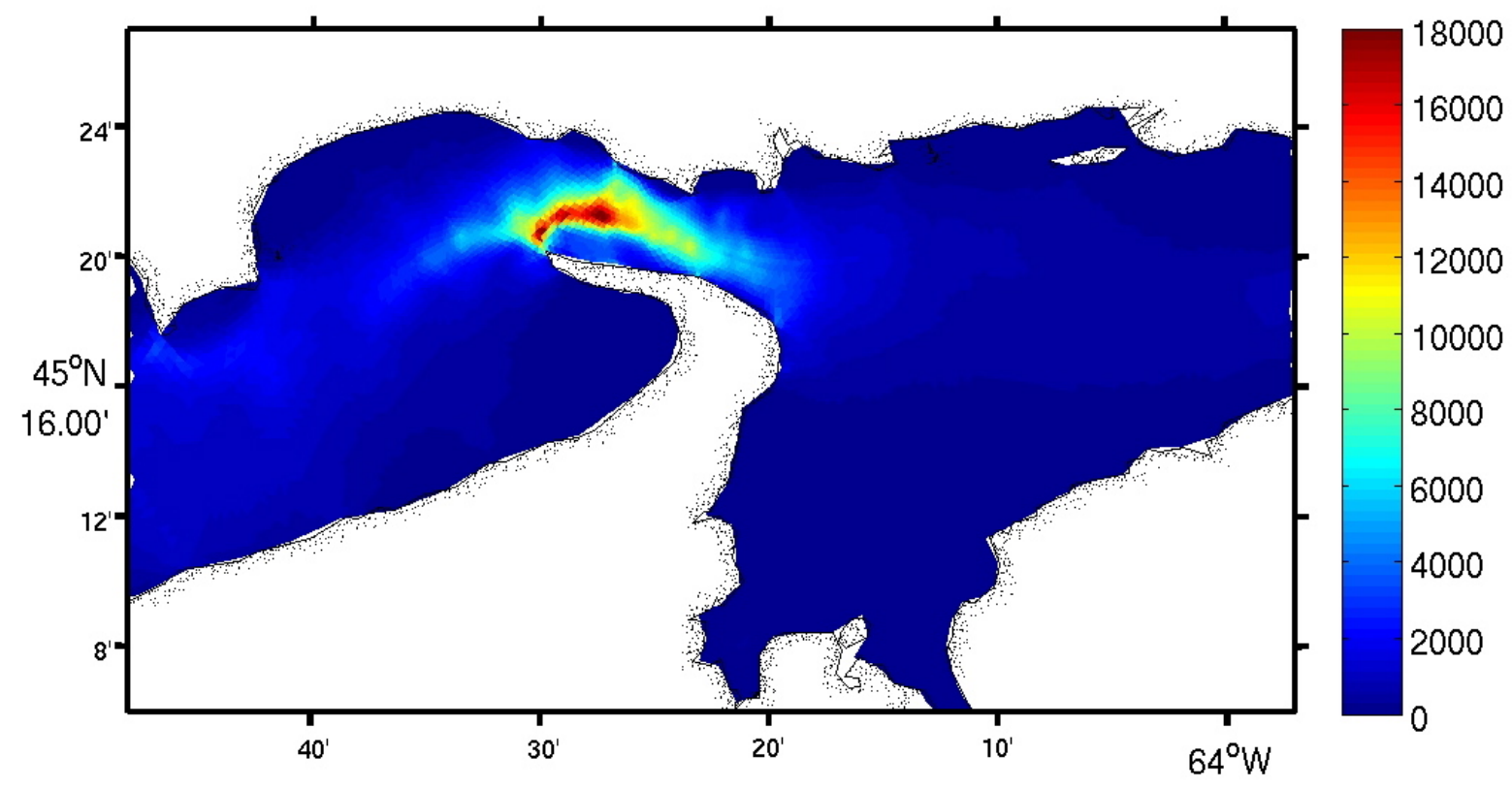

Figure 4: The power density $\left(\mathrm{kW} / \mathrm{m}^{2}\right)$ in the Minas Passage is much greater in this region than anywhere else in the Bay of Fundy - Gulf of Maine where the power density is typically less than $2 \mathrm{~kW} / \mathrm{m}^{2}$. The high power density in this region makes it a promising location for the implementation of turbines.

\section{Turbines and Tidal Power}

As stated above, the strongest currents in the Bay of Fundy are located in the Minas Passage, making it a promising location for the installation of turbines. If too many turbines are placed in the channel, the flow will be impeded by the increased drag, causing the power of the current to decrease. There is, therefore, a theoretical maximum to the amount of tidal power that can be harnessed. The theory presented here is summarized from the theoretical model of Garrett and Cummins in [6], where they examined a channel connecting a small bay to the open ocean. Blanchfield et al. extended this theory to 
include the effects of flow acceleration in [2]. This theoretical model was then adapted to the Minas Passage by Karsten et al. in [10].

To apply the theory of [6], we consider implementing turbines in the Minas Passage by introducing a drag force, $F_{d}$, to the flow between the small inner bay (Minas Basin) and the large exterior ocean (Bay of Fundy). The tidal elevation at the entrance to the Minas Passage is expressed as $a \cos \omega t$ and inside the Minas Basin as $\zeta_{b}(t)$ (see Figure 3). It is then assumed that there exists a balance between the turbine drag and the hydrostatic pressure gradient that results from the tidal head across the channel. This balance is expressed mathematically as follows,

$$
\zeta_{b}+\frac{L_{c}}{g} F_{d}=a \cos \omega t
$$

where $L_{c}$ is the length of the channel over which the turbines are located (see Figure 3). For the simplest analysis, it is assumed that the drag force is linear, that is $F_{d}=r_{1} u$, where $r_{1}$ is the frictional coefficient associated with the turbines and $u$ is the velocity of the current along the channel. Continuity can also be applied by assuming that the change in volume in the small bay is equal to the flux through the channel, resulting in the differential equation,

$$
A_{b} \frac{d \zeta_{b}}{d t}=E_{c} u
$$

where $A_{b}$ is the area of the small bay and $E_{c}$ is the cross-sectional area of the channel. Equations $(9)$ and $(10)$ can be derived from the governing shallow water equations $\sqrt{6}-$ 8) by integrating the momentum equations over the Minas Passage and the continuity equation over the Minas Basin, respectively. In both cases the non-linear effects are ignored.

Equation (10) can be solved for $u$ and then substituted into Equation (9) to give the following differential equation,

$$
\frac{\delta_{1}}{\omega} \frac{d \zeta_{b}}{d t}+\zeta_{b}=a \cos \omega t
$$

where

$$
\delta_{1}=\frac{r_{1} L_{c} A_{b} \omega}{g E_{c}} .
$$

This first order, linear differential equation can be solved to give

$$
\begin{gathered}
\zeta_{b}=\frac{a}{\sqrt{1+\delta_{1}^{2}}} \cos (\omega t-\phi), \\
\tan \phi=\delta_{1},
\end{gathered}
$$

where $\phi$ is the phase lag across the channel. Equation (11) can then be differentiated and substituted into Equation (10) to determine the velocity of the flow as

$$
u=-\frac{1}{\sqrt{1+\delta_{1}^{2}}} \frac{A_{b} \omega a}{E_{c}} \sin (\omega t-\phi) .
$$


The linear turbine drag, $F_{d}=r_{1} u$, can now be written as follows,

$$
F_{d}=-\frac{\delta_{1}}{\sqrt{1+\delta_{1}^{2}}} \frac{g a}{L_{c}} \sin (\omega t-\phi) .
$$

From this equation, it is evident that the magnitude of the drag force is an increasing function of $\delta_{1}$ that varies like

$$
\left|F_{d}\right| \sim \frac{\delta_{1}}{\sqrt{1+\delta_{1}^{2}}} .
$$

At large $\delta_{1}$, the fraction in Equation (15) approaches 1; therefore, at high friction, the magnitude of the drag force varies slowly and approaches a constant value. For small $\delta_{1}$, Equation 12 can be used to make the approximation, $\phi \approx \delta_{1}$. Equation (11) can also be used to conclude that the magnitude of the drag force at low friction varies like

$$
\left|F_{d}\right| \sim \phi \frac{\left|\zeta_{b}\right|}{a}
$$

which indicates that the force increases linearly with the increase in phase. Combining the approximations for high friction and low friction, it can be concluded that the drag force initially increases rapidly with $\delta_{1}$ before approaching a constant value at large $\delta_{1}$. On the other hand, the velocity, given by Equation (13), decreases slowly at first, and then rapidly at large $\delta_{1}$. The average extractable power is proportional to the product of $F_{d}$ and $u$, as follows,

$$
P=\frac{\rho E_{c} L_{c}}{T} \int_{0}^{T} F_{d} u d t .
$$

As $\delta_{1}$ is increased from zero, $F_{d}$ initially increases faster than $u$ decreases; therefore, at low friction the power rises quickly. Eventually, the phase, and hence $F_{d}$, reaches its maximum, but $u$ continues to decrease, causing the power to decrease towards zero. This behavior is evident in Figure 5, where the extractable power is plotted.

The exact function describing the power can be determined by substituting Equations (13) and (14) into Equation (17) and then integrating,

$$
P=\frac{1}{2}\left(\frac{\delta_{1}}{1+\delta_{1}^{2}}\right) \rho g A_{b} \omega a^{2} .
$$

It can be easily shown that $\delta_{1}=1$ corresponds to the maximum power, which allows the following simplification to be made,

$$
P=\frac{2 \delta_{1}}{1+\delta_{1}^{2}} P_{\max }
$$

where

$$
P_{\text {max }}=\frac{1}{4} \rho g A_{b} \omega a^{2} .
$$

Using the parameters summarized in Table 2, this linear theory provides an estimate of $P_{\max }=8.0 \mathrm{GW}$ for the Minas Passage.

In deriving Equation (18), it was assumed that the drag force was linear; however, it is more realistic to assume a quadratic drag force because the friction forces, $F_{x}$ and $F_{y}$, 


\begin{tabular}{|c|c|c|}
\hline Parameter & Description & Calculated Value \\
\hline$\rho$ & water density & $1026 \mathrm{~kg} / \mathrm{m}^{3}$ \\
$L_{c}$ & Length of Minas Passage & $1.2 \times 10^{4} \mathrm{~m}$ \\
$E_{c}$ & Cross-sectional area of Minas Passage & $3.1 \times 10^{5} \mathrm{~m}^{2}$ \\
$A_{b}$ & Surface area of Minas basin & $1.0 \times 10^{9} \mathrm{~m}^{2}$ \\
$\omega$ & $\mathrm{M}_{2}$ tidal frequency & $1.4 \times 10^{-4} \mathrm{~s}^{-1}$ \\
$a$ & amplitude of forcing tides & $4.73 \mathrm{~m}$ \\
\hline
\end{tabular}

Table 2: Parameters and their values for the Minas Passage and the Minas Basin.

are quadratic in the momentum equations (6) and 7). In [6] and [10], the drag force was represented by $F_{d}=r_{2} u|u|$. The analogous results to the quadratic theory of Karsten et al. [10] can be derived from our linear model by determining the appropriate quadratic drag parameter, $\delta_{2}$. The solution, $u$ and $\zeta$, (Equations 11 and 13 ) will also be a solution for quadratic drag if Equation (9) is satisfied; that is, if the drag force, $F_{d}$, is equivalent in the linear and quadratic cases. Thus,

$$
r_{1} u=r_{2} u|u|
$$

where $u$ is given by Equation (13), so

$$
\frac{\delta_{1}}{\sqrt{1+\delta_{1}^{2}}} \sin (\omega t-\phi)=\frac{\delta_{2}}{1+\delta_{1}^{2}} \sin (\omega t-\phi)|\sin (\omega t-\phi)|
$$

where

$$
\delta_{2}=\frac{r_{2} L_{c} A_{b}^{2} \omega^{2} a}{g E_{c}^{2}} .
$$

Equation (19) can be approximated by expanding the right hand side using a Fourier series as follows,

$$
\frac{\delta_{1}}{\sqrt{1+\delta_{1}^{2}}} \sin (\omega t-\phi)=\frac{\delta_{2}}{1+\delta_{1}^{2}}\left(\frac{8}{3 \pi} \sin (\omega t-\phi)-\frac{8}{15 \pi} \sin \left(3 \omega t-\phi_{3}\right)+\ldots\right) .
$$

It should be noted that the amplitude of the lowest order mode in the Fourier expansion is at least fives times larger than the amplitudes of the higher order modes. The frequency of the first term in the expansion is $\omega$, which corresponds to the frequency of the $\mathrm{M}_{2}$ tide; therefore, it can be concluded that the tides are dominated by the $\mathrm{M}_{2}$ constituent. Because their amplitudes are small, the higher order modes in Equation (20) can be neglected. The coefficients on both sides of the resulting equation can then be matched in order to determine the following relationship between $\delta_{1}$ and $\delta_{2}$,

$$
\delta_{2}=\frac{3 \pi}{8} \delta_{1} \sqrt{1+\delta_{1}^{2}}
$$

or conversely,

$$
\delta_{1}=\frac{8}{3 \pi} \delta_{2} \sqrt{\frac{2}{1+\sqrt{1+4\left(\frac{8}{3 \pi}\right)^{2} \delta_{2}^{2}}}} .
$$


By expressing $\delta_{1}$ in terms of $\delta_{2}$, the results of the linear theory can be transformed into the quadratic drag solutions of Karsten et al. [10] in the appropriate limit. In particular, the extracted power as a function of $\delta_{2}$ can be determined by substituting Equation (22) into Equation (18). This function is plotted in Figure 5 and it should be noted that the maximum extractable power occurs at $\delta_{2}=3 \sqrt{2} \pi / 8$ which corresponds to $\delta_{1}=1$ in Equation (21).

In order to compare the above theory to the results of simulations, it is necessary to model the effect that turbines would have on the tides. In essence, the turbines cause the water to slow down, acting as a drag on the flow. In a two-dimensional model, the simplest way to represent the increased drag is to augment the bottom friction, as was done in [13]. More specifically, the bottom friction is increased by an amount $\kappa_{t}$ at each of the nodes in the region defined by $L_{c}$ in Figure 3 . By simulating a turbine at each node in the Minas Passage, a "turbine farm" is essentially represented.

For each numerical simulation, the total bottom friction drag power, $D$, is determined by $D=\frac{1}{2} \rho\left(\kappa_{0}+\kappa_{t}\right)\left(u^{2}+v^{2}\right)^{3 / 2}$ where $\kappa_{0}$ is the natural bottom friction coefficient, 0.0026 . Figure 5 illustrates the agreement between the numerical simulations and the theory presented above. As expected, the power initially increases rapidly due to the phase lag, but eventually the decrease in speed causes the extracted power to decrease. Although it is not so evident from Figure 5, in the limit as $\delta_{2} \rightarrow \infty, P \rightarrow 0$ for both the theory and the numerical simulations.

The theory presented here has several limitations. For simplicity, it does not contain the effects of the acceleration or the nonlinearity of the flow. As well, the natural bottom friction is not separated from the turbine friction in the calculations of the extractable power. These issues are addressed in Karsten et al. [10, resulting in a better comparison of the theoretical and numerical results. As is evident in Figure 5, the quadratic theory overestimates the maximum extractable power at high friction. The numerical simulations suggest that the maximum drag power in the Minas Passage is approximately $7.3 \mathrm{GW}$. The power associated with only the turbine friction can be approximated by,

$$
P_{t}=\frac{\kappa_{t}}{\kappa_{t}+\kappa_{0}} D
$$

At maximum frictional power $\kappa_{t}=0.05$ and $D=7.3 \mathrm{GW}$, thus it can be estimated that up to $6.9 \mathrm{GW}$ of power can be extracted by the turbines.

Obviously, when energy is removed from a system, especially a system governed by resonance, there are bound to be effects on the areas both near and far from the power extraction site. Figure 6 plots the relative change in tidal amplitude if the maximum power is extracted. Because the flow through the Minas Passage is restricted by the presence of the turbines, the amplitude of the tide within the Minas Basin decreases (Equation 11). It would also make sense for the tides to decrease everywhere in the region, since energy is being removed; however, this is not evident from the simulations (see Figure 6) as the tides actually increase by 10 to $15 \%$ throughout the Gulf of Maine.

As mentioned earlier, the Bay of Fundy is characterized by high tides due to the resonance that results because the length of the bay is nearly equal to one quarter of the wavelength of the tides. By adding turbines, the flow through the Minas Passage takes longer to reach the Minas Basin, which implies that the system is moving away from resonance; however, the turbines also cause some of the water to no longer enter the 


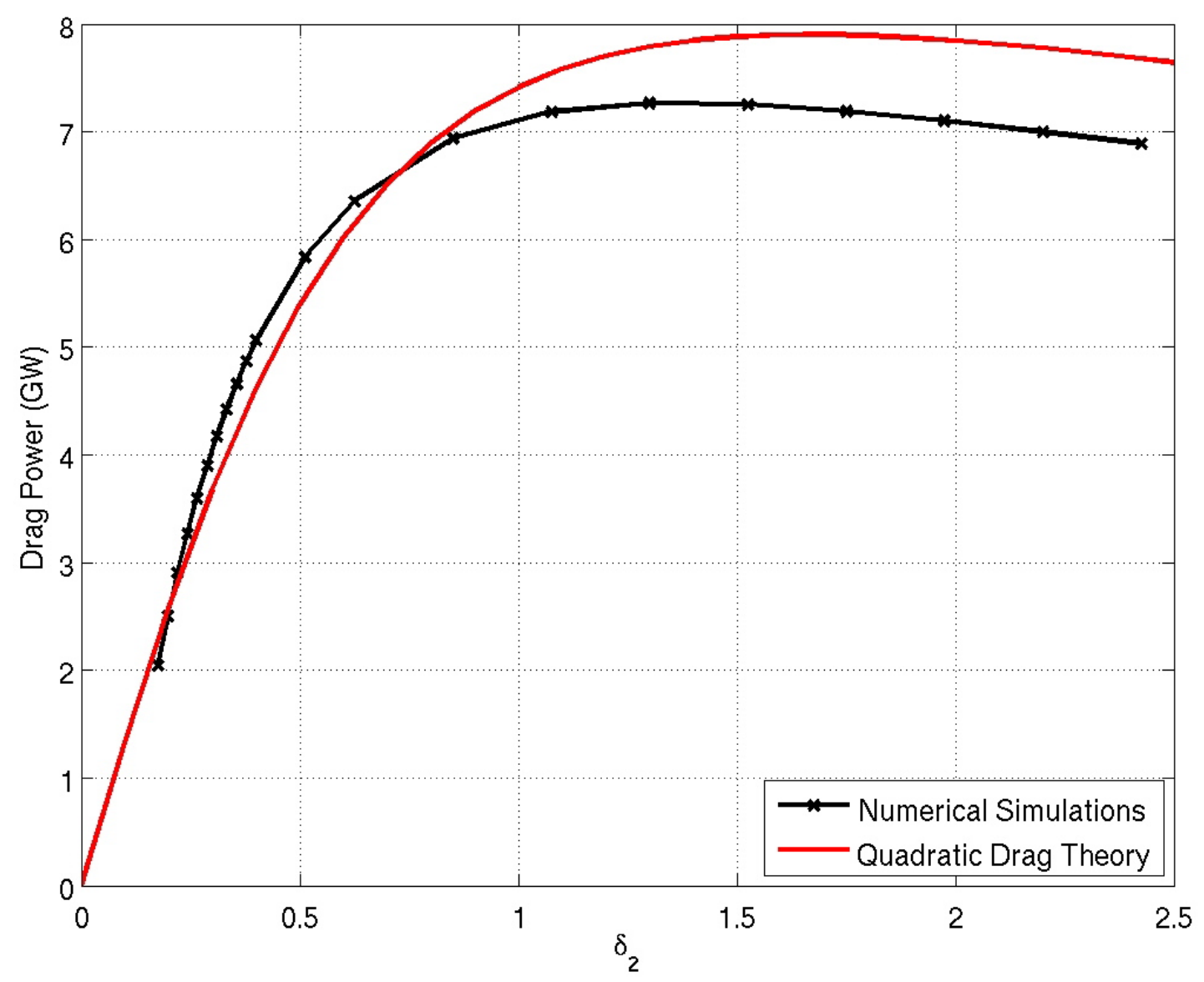

Figure 5: A comparison between the frictional power values as determined by both theory and numerical results. Each ' $\mathrm{x}$ ' marker corresponds to a particular simulation that was completed.

Minas Passage, shortening the bay and causing the system to move closer to resonance. Due to these contrasting arguments it is not immediately clear whether the turbines cause the system to move towards or away from resonance. In [10], numerical simulations were performed to calculate the resonant period of the system. This was done by varying the period of the forcing tides until the total energy in the system was a maximum. Using this method, Karsten et al. [10] calculated a period of 12.85 hours for the undisturbed system. The results of [10] indicate that the resonant period of the system decreases as the turbine drag is increased. In particular, a period of 12.80 hours was calculated for weak turbines $\left(\kappa_{t}=0.005\right)$, whereas, at maximum turbine power $\left(\kappa_{t}=0.05\right)$, the period was reduced to 12.59 hours. The placement of a barrier across the Minas Passage caused the system to move even closer to resonance with a period of 12.50 hours. Because the natural period of 12.42 hours is being approached as the turbine drag increases, stronger resonance should amplify the tidal amplitudes. For the simulations, this is true throughout the Gulf of Maine (see Figure 6).

The amplitude and phase changes that occur at nine locations are summarized in Table 3 for a low friction simulation, a high friction simulation and a simulation with a 


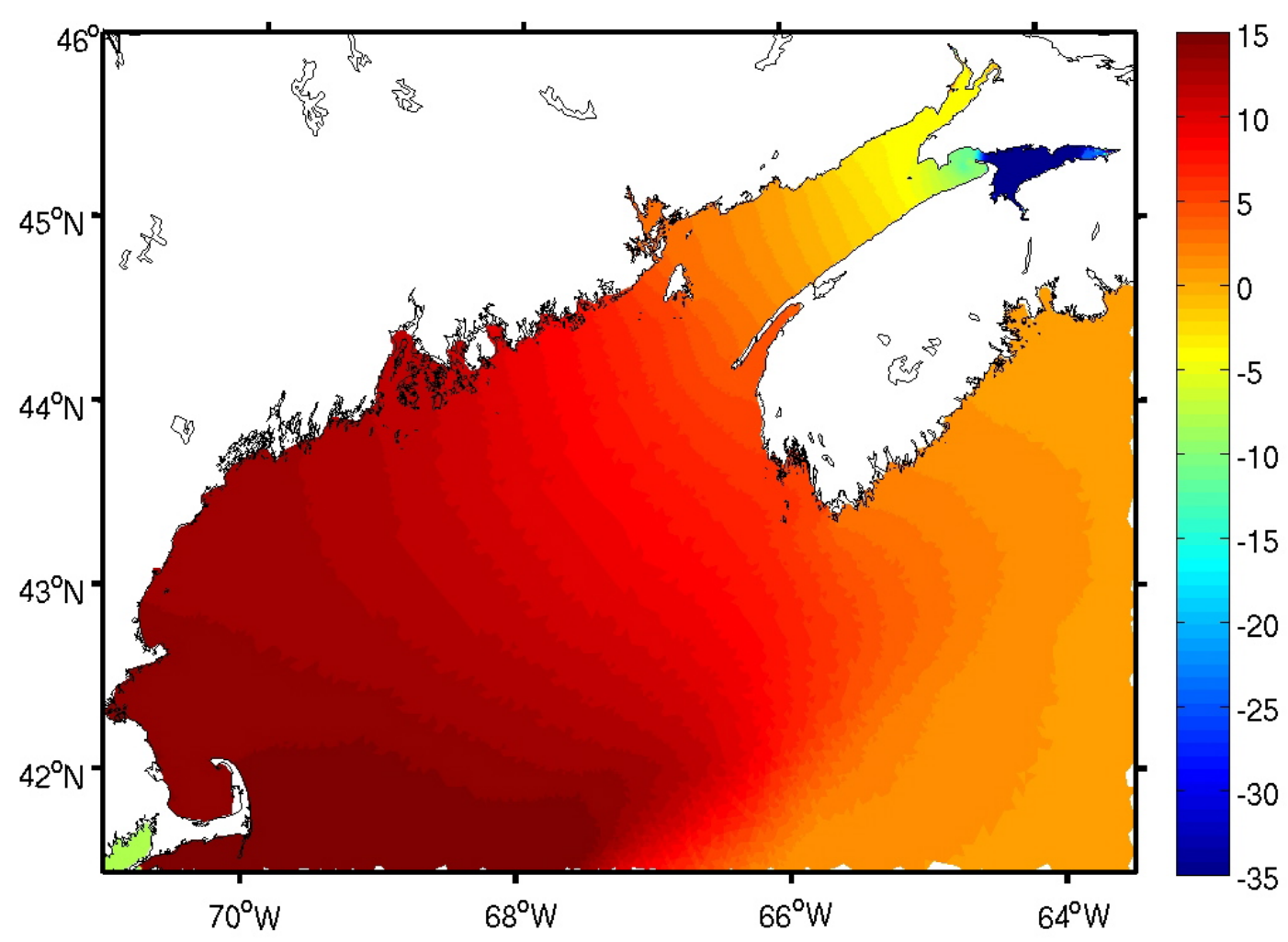

Figure 6: The relative change in amplitude (\%) of the tides as a result of extracting the maximum amount of power from the Minas Passage.

barrier placed across the Minas Passage. Previous studies, 9] and [12, have examined the barrier case in greater detail. The results of the simulations suggest that both implementing a barrier and extracting the maximum power would lead to significant effects on the tides throughout the entire region. The greatest effects occur in the Minas Basin where extracting the maximum amount of power would cause the amplitude of the tides to decrease by $195 \mathrm{~cm}$. The natural tides at this location are $548 \mathrm{~cm}$ (Table 1), therefore, the simulations indicate that the tidal amplitude is reduced by $36 \%$. In addition, the simulations suggest that the phase lag between Cape Split and the Minas Basin increases by $34^{\circ}$ (see Table 3), resulting in a total phase difference of $44^{\circ}$ across the Minas Passage. The theory presented above gives $\delta_{1}=1$ at maximum power; therefore, Equations (11) and (12) suggest a 30\% reduction in tidal amplitude in the Minas Basin, in addition to a phase lag of $45^{\circ}$. At lower friction $\left(\kappa_{t}=0.005\right) \delta_{2}=0.29$; therefore, by Equation (21), $\delta_{1}=0.24$. This can then be used to predict a $3 \%$ reduction in tidal amplitude and a phase lag of $13^{\circ}$. As Table 3 indicates, the numerical simulations give a $31 \mathrm{~cm}$, or $6 \%$, reduction in tidal amplitude. The numerical simulations also result in a $7^{\circ}$ increase in phase lag between Cape Split and the Minas Basin, which corresponds to a total phase lag of $17^{\circ}$. Considering the limitations of this theory, the agreement between it and the simulations is excellent. By including the effects of acceleration and the nonlinearity of the flow, Karsten et al. [10] obtained better agreement between their theory and simulations. Overall, it is 
important to note that extracting maximum power would have a significant effect on the tides; however, approximately $2.5 \mathrm{GW}\left(\kappa_{t}=0.005\right)$ could be extracted by turbines with less than a $6 \%$ change in tidal amplitude throughout the Bay of Fundy - Gulf of Maine region. It is unlikely that such a small change in the tides would have significant effects on the environmental aspects of the region, and yet a large amount of power could still be harnessed.

\begin{tabular}{|c|ccc|ccc|}
\hline & \multicolumn{3}{|c|}{ Amplitude Change $(\mathrm{cm})$} & \multicolumn{3}{c|}{ Phase Change $\left(^{\circ}\right)$} \\
\cline { 2 - 6 } Station & $\kappa_{t}=0.005$ & $\kappa_{t}=0.05$ & barrier & $\kappa_{t}=0.005$ & $\kappa_{t}=0.05$ & barrier \\
\hline Boston & 3 & 19 & 47 & -1.6 & -4.7 & -0.6 \\
Portland & 3 & 18 & 45 & -1.6 & -4.9 & -1.4 \\
Saint John & -3 & 1 & 41 & -1.8 & -8.8 & -13.8 \\
Chignecto & -8 & -18 & 27 & -1.8 & -9.9 & -18.2 \\
Minas Basin & -31 & -195 & - & 4.3 & 20.7 & - \\
Cape Split & -16 & -55 & -30 & -2.6 & -13.8 & -31.0 \\
Isle Haute & -7 & -20 & 16 & -1.7 & -9.6 & -18.5 \\
Westport & 0 & 9 & 35 & -1.5 & -6.3 & -7.5 \\
Yarmouth & 1 & 9 & 26 & -1.2 & -4.2 & -3.2 \\
\hline
\end{tabular}

Table 3: The changes in phase and amplitude resulting from a low friction simulation $\left(\kappa_{t}=0.005\right)$, a high friction simulation $\left(\kappa_{t}=0.05\right)$ and a simulation with a barrier placed across the Minas Passage. The extracted turbine power for the low friction and high friction simulations are $2.5 \mathrm{GW}$ and $6.9 \mathrm{GW}$, respectively.

\section{Conclusion}

The present demand for renewable energy has led to the discussion and investigation of tidal power in the Bay of Fundy. With the highest tides in the world, it is obvious why this region is of particular interest. Numerical simulations and a simple one-dimensional theory were both used to approximate the natural period of the Bay of Fundy as 12.85 and 13.5 hours, respectively. Although both of these estimates are slightly higher than the 12.42 hour period of the $\mathrm{M}_{2}$ tide, the near-resonance causes large tidal amplitudes to occur in the Minas Basin.

The tides throughout the Bay of Fundy and Gulf of Maine were accurately simulated using a numerical model. The results of these simulations confirmed that the fastest currents are located in the Minas Passage, making it a promising location for the implementation of turbines.

By increasing the bottom friction in the Minas Passage, numerical simulations were completed that examined the effect of turbines on the tides. In agreement with a simple theory, it was estimated that up to $6.9 \mathrm{GW}$ of power is available for extraction. The theory and simulations also suggest that extracting maximum power would have a significant effect (36\% reduction) on the Minas Basin tides. Furthermore, simulations indicated that the entire system would move closer to resonance and thus cause the tides in Boston to increase by $15 \%$. A change of this magnitude would most likely have serious environmental consequences; however, because the power at low resistance is driven by the phase lag 
across the Minas Passage, up to $2.5 \mathrm{GW}$ of power could be extracted with less than a $6 \%$ change on the tides both locally and afar.

Through this research, it can therefore be concluded that a significant amount of power can be extracted from the Minas Passage with minimal consequences on the tides. This encourages the continued discussion of tidal power in the region. Further research would involve performing more detailed numerical simulations where the turbines are more accurately modeled to examine the resulting changes in flow patterns. The objective of further research is to determine the most efficient and ecologically acceptable placement of turbines.

\section{Acknowledgments}

The authors thank their supervisors, Richard Karsten and Ronald Haynes, for their guidance in conducting this research and in preparing this paper. As well, we would like to thank David Greenberg for providing the data for the numerical grid and observations. The comments of the two anonymous reviewers are also appreciated as they helped to significantly improve this paper. Finally, thanks must be extended to the Natural Sciences and Engineering Research Council for the financial support that it provided both authors through USRA awards.

\section{References}

[1] R. Bishop, Tides and the Earth-Moon System, Observer's Handbook 2008, (2007), p. 173.

[2] J. Blanchfield, C. Garrett, P. Wild, and A. Rowe, The Extractable Power from a Channel Linking a Bay to the Open Ocean, Proceedings of the Institution of Mechanical Engineers, Part A: Journal of Power and Energy, 222 (2008), pp. 289-297.

[3] C. Chen, R. Benrdsley, And G. Cowles, An unstructured grid, finite-volume coastal ocean model (FVCOM) system. Special Issue entitled "Advance in Computational Oceanography", Oceanography, 19 (2006), pp. 78-89.

[4] F. Dupont, C. Hannah, And D. Greenberg, Modelling the Sea Level of the Upper Bay of Fundy, Atmosphere-Ocean, 43 (2005), pp. 33-47.

[5] C. Garrett, Tidal Resonance in the Bay of Fundy and Gulf of Maine, Nature, 238 (1972), pp. 441-443.

[6] C. Garrett and P. Cummins, Generating Power from Tidal Currents, Journal of Waterway, Port, Coastal and Ocean, 130 (2004), pp. 114-118.

[7] C. Garrett and P. Cummins, The Power Potential of Tidal Currents in Channels, Proceedings of The Royal Society, 461 (2005), pp. 2563-2572.

[8] A. Gill, Atmosphere-Ocean Dynamics, Academic Press, San Diego, CA, 1982. 
[9] D. Greenberg, A numerical model investigation of tidal phenomena in the Bay of Fundy and Gulf of Maine, Marine Geodesy, 2 (1979), pp. 161-187.

[10] R. Karsten, J. McMillan, M. Lickley, and R. Haynes, Assessment of Tidal Current Energy for the Minas Passage, Bay of Fundy, Proceedings of the Institution of Mechanical Engineers, Part A: Journal of Power and Energy, in press (2008).

[11] Nova Scotia to create test centre for tidal power, The Canadian Press (2008, January 8), The Globe and Mail.

[12] P. Sucsy, B. Pearce, And V. Panchang, Comparison of Two-and ThreeDimensional Model Simulation of the Effect of a Tidal Barrier on the Gulf of Maine Tides, Journal of Physical Oceanography, 23 (2006), pp. 1231-1248.

[13] G. Sutherland, M. Foreman, and C. Garrett, Tidal current energy assessment for Johnstone Strait, Vancouver Island, Proceedings of the Institution of Mechanical Engineers, Part A: Journal of Power and Energy, 221 (2007), pp. 147-157.

[14] Triton Consultants Ltd., Canada Ocean Energy Atlas (Phase 1) Potential Tidal Current Energy Resources Analysis Background, 2006. Available at http://homepage.mac.com/max.larson/Triton/Papers.html. 